\title{
Urostomy Obstruction
}

National Cancer Institute

\section{Source}

National Cancer Institute. Urostomy Obstruction. NCI Thesaurus. Code C78680.

Blockage of the urostomy. 